\title{
Ankle range of motion and dynamic balance in recreational Sepak Takraw players with and without ankle injury - a comparative study
}

\author{
Jessica Phan Zhi Xin ${ }^{1 \mathrm{ABCDE}}$, Rajkumar Krishnan Vasanthi ${ }^{1,2 \mathrm{ABCD}}$, Vinoshkumar Purushothaman ${ }^{1 \mathrm{ACD}}$, \\ Ali Md. Nadzalan ${ }^{2 A C D}$ \\ ${ }^{1}$ Faculty of Health and Life Sciences, INTI International University, Nilai, Malaysia \\ ${ }^{2}$ Faculty of Sports Science and Coaching, Sultan Idris Education University, Tanjong Malim, Malaysia
}

\begin{abstract}
Background Sepak Takraw (ST) is considered Malaysia's national sport, and the nature of this sport requires players to and Study Aim be sufficiently acrobatic. Therefore, players were expected to jump and regain their balance, challenging their dynamic balance (DB). Nonetheless, range of motion (ROM) is closely related to balance. Hence, this study aimed to compare recreational ST players' ankle ROM and DB differences with and without ankle injuries.

Material and The cross-sectional study in which 30 participants were assigned purposively according to players with an Methods ankle injury $(n=15)$ and without ankle injury $(n=15)$ based on the position statement of the International Ankle Consortium (IAC). One time ankle Range of Motion and Dynamic balance was measured using a universal goniometer and Star Excursion Balance Test (SEBT), respectively, for both groups.

Results Independent T-test was performed to identify the significant differences $(p<0.05)$ of SEBT and ROM between the non-injured leg and injured leg within the injured group to the non-injured group. Ankle eversion and dorsiflexion between the injured and non-injured groups did not show any significant difference with $p=0.35 \& p=0.53$, respectively. As for SEBT, only posteromedial and medial of the injured leg did not show a significant difference $p>0.05$. All other ankles ROM and the SEBT score demonstrated a statistically significant difference $\mathrm{p}<0.05$.

Conclusions: $\quad$ ROM and DB training/rehabilitation should be incorporated to all the injured group ST sports players regardless of which leg is affected to optimize ankle function and the game performance.

Keywords: $\quad$ athletics injuries, team sports, sports, range of motion, postural balance
\end{abstract}

\section{Introduction}

Sepak Takraw (ST) is considered Malaysia's national sport, and it has the combination of few elements from other sports such as soccer, volleyball, baseball, badminton, and the ancient sport of Sepak Raga [1]. It is a sport widely known in South-East Asia and has transpired to other countries worldwide [2]. Sepak Takraw is a game where the ability to kick and juggle the ball around with the acrobatic moves of gymnasts is required. Lower extremities are used instead of upper extremities, and as in a volleyball, there are passes, sets and spikes in play. However, players can utilize their head, chest, feet and thighs to propel the ball over the net. Whether a player is a server, spiker, nor feeder, they ought to have good aerial agility with precision, leg strength, timing and skills [3].

Players are expected to jump and regain their balance, which is a challenge to their dynamic balance (DB) [2]. Nonetheless, range of motion (ROM) is closely related to balance that stretching could improve dynamic balance in athletics. There is only one known study done for Sepak Takraw, which was conducted in Japan to investigate injuries sustained with physical characteristics. In a study by Kubo et al [4] the ankle strain as the second-highest injury sustained, whereas the hamstring strain injury was the highest. Sprains are the recurrent injuries in high-risk sports, up to $80 \%$. (c) Jessica Phan Zhi Xin, Rajkumar Krishnan Vasanthi, Vinoshkumar Purushothaman, Ali Md. Nadzalan, 2021 doi:10.15561/26649837.2021.0603
Aside from sportspeople, an initial ankle sprain affects around $8 \%$ of the population. Those with a history of sprains suffer from residual or chronic discomfort, recurrent sprains, and perceived instability [5]. In the ankle joint, ankle sprain is the most recurrent injury that occurred which $20 \%$ of it will develop into chronic ankle instability (CAI) [6]. Thus, CAI became the interest of this research in defining 'Injured' ST players.

A study [7] mentioned that specific ROM where dorsiflexion influences the DB in individuals with CAI, which subsequently established difficulty in performing a portion of the Star Excursion Balance Test (SEBT). However, the authors stated that measures of ROM at other joints were not obtained, which confirms the need to further study it in other joint ROM of the ankle besides dorsiflexion, which are eversion, inversion, and plantarflexion. Looking deeper, a decrease in ankle ROM declines the balance control function on the ankle strategies [8]. However, there is a decline in DB and ROM through observation of dorsiflexion in individuals with CAI [9].

The investigation was done in integrating these data into this sport; ST players can benefit from revising a training regimen, rehabilitation programs for those who have CAI problems, and reducing or avoiding the incidence of recurrence of an ankle injury. Hence, this study compares ankle ROM and DB in recreational Sepak Takraw players with and without ankle injury (CAI). 


\section{Materials and Methods}

\section{Participants}

In this cross-sectional study, participants $(n=30)$ with a mean age of $24 \pm 4.03$ in non-injured group and $24.9 \pm 3.81$ from injured group were recruited and informed about the study and obtained written consent. The faculty Ethical Committee of the institution has (FHLS/RAC/ JUL/6) approved this study.

Based on the International Ankle Consortium (IAC) where the injured group should have at least have one significant history of ankle sprain as follows: "the initial sprain must happen at least 12 months before, have inflammatory symptoms (pain, swelling, etc.), at least one interrupted day of desired physical activity, the latest injury must happen more than three months before study enrolment and history of the previously injured ankle joint "giving way" and recurrent sprain and "feelings of instability where the participants ought to have at least two reports of giving way in the six months before study enrolment". Participants with a history of previous surgeries, fractures in either limb of the lower extremity, musculoskeletal injuries of the lower extremity in the past three months, which impacted joint integrity and function (i.e., sprains, fractures) that caused at least one interrupted day of desired physical activity and had bilateral CAI was excluded.

In our study, the participants from the non-injured group were excluded if they had a history of ankle sprain, fractures and past surgeries in both the lower extremity and acute musculoskeletal injuries of other joints in both the lower extremity in the past three months.

Procedure

The independent variables used in this study are the subjects, with or without any injuries (CAI), and the dependent variables are the ankle ROM and DB. Once the participants were concerned to participate in the study, they were categorized into the 'injured' group, which was further divided into 'injured leg' and 'non-injured leg' and non-injured group before quantitative measurement of ROM and SEBT.

\section{Ankle Range of Motion (ROM)}

Participants were put into a long sitting position, knee slightly flexed to $20^{\circ}[10]$ and ankle in a neutral position on the ground. The universal goniometer was the tool used to measure ankle ROM, and the lateral malleolus was the fulcrum, whereas the stationary arm was parallel to the fibula and tibia. The moving arm follows the 5th metatarsal. The starting position for the ankle is in a neutral position. Participants were asked to plantarflex and dorsiflex the ankle without moving the toes to control the toes contribution. Participants must return to the initial position before taking another reading. Three readings are taken each, and the average records were utilized [11].

Participants were asked to sit on high sitting to measure eversion and inversion. Between both malleoli, the anterior part of the ankle was the fulcrum; the stationary arm was parallel to the tibia and the moving arm follows the 2nd metatarsal. Similar, the starting position for the ankle is in a neutral position. However, this time, the toe contribution is not controlled. The initial position was returned, and the second measurement is taken. Recordings of three readings taken and then averaged out [12]. The data for both left and right ankle ROM were retrieved.

Dynamic Balance - Star Excursion Balance Test (SEBT)

SEBT was used to measure the dynamic balance of the participants. With hands-on the hip, participant's squat with one leg and the non-stance limb lightly touches one point along with all eight directions without shifting weight or rest on the ground at any given time during the test. The 8 points of direction - anterior, anteromedial, anterolateral, medial, lateral, posterior, posteromedial, and posterolateral challenge the DB of participants. Participants are not allowed to return to the center point during the test. The furthest point that participants could reach were marked and measured using a measuring tape. Participants that failed to follow these stipulations were disqualified. Five minutes of warm-up in their intensity level and four practice attempts were allowed before the actual recordings. Three records will be taken with 5 seconds of rest between each reach were given [7]. The data for both left and right stance retrieved.

Statistical Analysis:

Quantitative data of SEBT and ankle ROM of each player was measured and analyzed using SPSS version 23. Descriptive statistics are portrayed as mean \pm standard deviation. Since it is normal distribution data, we used an independent t-test to compare the differences of SEBT and ROM within the non-injured and injured leg of the injured group and between the injured and non-injured group. A p-value of $<0.05$ was considered statistically significant in this study.

\section{Results}

Twenty recreational Sepak Takraw male players volunteered to participate in this research. Among the fifteen participants under the injured group, eleven of the players had CAI on their right leg whereas four had it on their left leg (Table 1).

Table 1. Demographics of the participants

\begin{tabular}{lll}
\hline Groups & $\begin{array}{l}\text { Non-Injured } \\
\text { group }\end{array}$ & $\begin{array}{l}\text { Injured } \\
\text { group }\end{array}$ \\
\hline Participants & $\mathrm{n}=15$ & $\mathrm{n}=15$ \\
Age (mean \pm SD) & $24 \pm 4.03$ & $24.9 \pm 3.81$ \\
Height $(\mathrm{cm})$ (mean \pm SD) & $173.1 \pm 6.40$ & $169.4 \pm 5.27$ \\
\hline
\end{tabular}

\section{SD-Standard Deviation}

In Table 2, injured group had an increase in the dorsiflexion, plantarflexion and inversion except for eversion had a decrease ROM comparing to the Noninjured group.

Comparing the Injured leg within the injured group to the non-injured group exhibit no significant difference $(p>0.05)$ in dorsiflexion and eversion (Table 3$)$. This outcome also surfaced similar result while comparing the 
Table 2. Comparison of ROM degree (Mean \pm SD) within Injured group (Injured leg \& non-injured leg) with Non-injured group

\begin{tabular}{lllll}
\hline Motions & Dorsiflexion & Plantarflexion & Inversion & Eversion \\
\hline Injured Group Injured leg & $28.6 \pm 4.51^{*}$ & $33.13 \pm 8.62^{*}$ & $32.4 \pm 5.11^{*}$ & $9.9 \pm 3.55^{*}$ \\
Injured Group Non-injured leg & $29.3 \pm 4.37^{*}$ & $29.47 \pm 7.9^{*}$ & $32.93 \pm 6.11^{*}$ & $10.17 \pm 5.03^{*}$ \\
Non-injured Group & $24.15 \pm 5.56^{*}$ & $22.33 \pm 2.77^{*}$ & $22.72 \pm 4.06^{*}$ & $11.4 \pm 3.55^{*}$ \\
\hline
\end{tabular}

*Mean \pm SD of ROM in degrees, SD-Standard Deviation

Table 3. Significance level of ROM within Injured group (injured leg \& non-injured leg) with Non-injured group

\begin{tabular}{lllll} 
Motions & Dorsiflexion & Plantarflexion & Inversion & Eversion \\
Injured leg vs Non-injured group & $0.066^{\dagger}$ & $0.003^{*}$ & $0.000^{*}$ & $0.357^{\dagger}$ \\
Non-injured leg vs Non-injured group & $0.034^{*}$ & $0.021^{*}$ & $0.000^{*}$ & $0.535^{+}$ \\
\hline${ }^{*} \mathrm{p}<0.05^{+} \mathrm{p}>0.05$ & & &
\end{tabular}

Table 4. Comparison of SEBT (Mean \pm SD) within Injured group (Injured leg \& non-injured leg) with Non-injured group.

\begin{tabular}{|c|c|c|c|c|c|c|c|c|}
\hline Directions & Ant & Ant.L & Lat & Post.L & Post & Post.M & Med & Ant.M \\
\hline $\begin{array}{l}\text { Injured Group } \\
\text { Injured leg }\end{array}$ & $84.87 \pm 8.22^{*}$ & $76.4 \pm 13.39^{*}$ & $73.67 \pm 6.91^{*}$ & $72.63 \pm 9.99^{*}$ & $70.27 \pm 9.3^{*}$ & $68.33 \pm 10.21^{*}$ & ${ }^{*} 69.4 \pm 12.1^{*}$ & $66.97 \pm 11.42^{*}$ \\
\hline $\begin{array}{l}\text { Injured Group } \\
\text { Non-injured leg }\end{array}$ & $85 \pm 8.1^{*}$ & $76.93 \pm 15.57^{*}$ & ${ }^{*} 73.77 \pm 4.4^{*}$ & $75 \pm 9.2^{*}$ & $69.6 \pm 9.06^{*}$ & $64.97 \pm 10.7^{*}$ & $63.9 \pm 7.35^{*}$ & $66.9 \pm 11.63^{*}$ \\
\hline $\begin{array}{l}\text { Non-injured } \\
\text { Group }\end{array}$ & $93.77 \pm 3.9^{*}$ & $93.55 \pm 4.88^{*}$ & $90.05 \pm 5.19^{*}$ & $83.6 \pm 5.29^{*}$ & $79.63 \pm 5.62^{*}$ & $73.17 \pm 3.43^{*}$ & $77.12 \pm 3.71^{*}$ & $84.28 \pm 2.86^{*}$ \\
\hline
\end{tabular}

Table 5. Significance level of SEBT within Injured group (injured leg \& non-injured leg) with Non-injured group.

\begin{tabular}{lllllllll}
\hline Directions & Ant & Ant.L & Lat & Post.L & Post & Post.M & Med & Ant.M \\
\hline $\begin{array}{l}\text { Injured leg vs Non- } \\
\text { injured group }\end{array}$ & $0.009^{*}$ & $0.003^{*}$ & $0.000^{*}$ & $0.009^{*}$ & $0.016^{*}$ & $0.184^{+}$ & $0.081^{+}$ & $0.001^{*}$ \\
$\begin{array}{l}\text { Non-injured leg vs } \\
\text { Non-injured group }\end{array}$ & $0.009^{*}$ & $0.008^{*}$ & $0.000^{*}$ & $0.022^{*}$ & $0.009^{*}$ & $0.042^{*}$ & $0.000^{*}$ & $0.001^{*}$ \\
\hline
\end{tabular}

${ }^{*} p<0.05,{ }^{\dagger} p>0.05$ Ant-Anterior, Ant.L-Anteriolateral, Lat-Lateral, Post.L -Posteriolateral, Post-Posterior, Med-Medial, Ant.M-Ateriomedial.

eversion ROM of non-injured leg within the injured group to the non-injured group (Table 3).

In table 4 , the non-injured group were able to reach further than the injured group in SEB test. Posteromedial plane and Medial plane SEBT gave a result of no significant difference (Table 5).

\section{Discussion}

Our study indicates that there were significant differences in the mean \pm SD of ankle ROM in all planes. Excessive ROM was observed in ankle dorsiflexion, plantarflexion and inversion in the injured leg compared to the non-injured group, and this is suggestive of ankle hypermobility in the injured group. Nevertheless, profoundly reduced the amount of ankle eversion ROM compared to the other movements in the injured leg. In contrast, the previous study results observed a decrease in the dorsiflexion range in the CAI population [13-15]. The current study found that there is gross hypermobility in ROM in all planes in the injured leg. The inversion joint laxity, anterior joint laxity, increased tibiotalar anterior translation and subtalar hypermobility following CAI was the potential cause for this hypermobility [16, 17]. Interestingly, the present study detected a more significant difference in inversion ROM in both the injured and noninjured groups. This report coincides with the statement made in the earlier study that a more substantial amount of inversion and plantar flexion of the foot to tibia was noted in the CAI population compared to healthy subjects $[18,19]$.

Furthermore, the result of this study provides a fascinating report that the ROM in the non-injured leg and non-injured group also has dramatic significance except for eversion. The possible rationale for these findings aligns with the former study that people with CAI would have poor postural, neuromuscular control and compromised 
proprioception. Additional literature also attests that CAI affects both the injured and uninjured ankles due to the disrupted sensorimotor system [20, 21]. Earlier investigations affirm no significant difference between ankle dorsiflexion in subjects with CAI compared to the coper or control group [22]. This exposition concurs with the current study regarding dorsiflexion ROM between the injured leg and the non-injured group. The disparity findings in this study regarding ankle dorsiflexion ROM in the non-injured leg within the injured group raise a recommendation for further research to comprehend the reason behind this phenomenon. Additionally, this research also bestows no effect on ankle eversion range regardless of CAI or otherwise. On the other hand, ankle plantarflexion and ankle inversion showed significant differences; thus, rehabilitation should focus on these two planes regardless of the injured leg.

The current study found that the dynamic balance in posterior, posterior medial and medial excursion of the injured leg shows lesser reading than the non-injured leg in the injury group. However, this study result differed from the previous investigation in which identified excursion deficit was only in the posterior medial direction in subjects with CAI [15]. On the contrary, the previous study also observed that anterior reach was more affected but not the posterior medial and Posterior lateral reaches because mechanical restrictions would alter the anterior spread while the posterior medial and Posterior lateral depends on the strength and postural control in SEBT [23].

In this research, the overall non-injured group had a better dynamic balance when compared to the injured group at all distances. The extent of our study also declares that there is a significant difference in dynamic balance in all lengths except in posterior medial and medial directions between an injured leg and a non-injured group. Although there is no significance in posterior medial and medial distances, dynamic balance of the injured leg in anterior, anterolateral, lateral, posterior and posterolateral directions shows greater significance than the non-injured leg. Similar to earlier literature, where the outcome showed that the injured leg reached significantly less when compared to the non-injured leg or noninjured group [14, 24, 25]. In addition, the same pattern of findings was observed while examining the dynamic balance between football and basket players with CAI in which the SEBT shows a significant difference between injured and non-injured leg in players [26].
In our study, we also reported that posteromedial and medial plane had no significance ( $\mathrm{p}$-value 0.184 and 0.081 respectively) between the injured leg and non-injured group, it is contrasted with the findings from the former study that injured leg had a significant difference in all the planes of SEBT in CAI subjects [25]. Subsequently, another investigation demonstrated that the bilateral impairment of proprioception accompanies unilateral ankle instability is evident [27]. This result also corroborates our findings, in which there is a substantial significance in dynamic balance in all planes between the non-injured leg and the non-injured group. The potential source of the symptom is from the adjustment in the central nervous system that appears as a neuromuscular deficit in the uninjured lower extremity joints [28].

In the future, research with a large population considers other contributing factors that may affect the SEBT performance, such as proprioception and muscle strength in the lower limb in Sepak Takraw players. Next, this study is limited to 18 to 30 years old, which would broaden to investigate other age groups. Irrespective of the injured or non-injured leg, the inversion and plantar flexion ROM is more significant between the injured and non-injured groups. Hence, future studies can analyze the factors influencing the impaired ROM in the normal leg in these players to prevent further injury in the proximal joints of both lower extremities. It is established that CAI players have a considerable difference when contrast to the non-injured group in terms of ROM and DB. Thus, regardless of which leg is affected, both limbs should endorse ROM, DB or multicomponent training/ rehabilitation to improve ankle function [29].

\section{Conclusions}

Ankle plantarflexion and inversion exhibited significant difference in injured group versus non-injured group. However, only ankle dorsiflexion from the noninjured leg showed the same as well. SEBT revealed that all planes had significant difference in injured group versus non-injured group except for posteromedial and medial of the injured leg. ROM and DB training/rehabilitation should be done on the injured group regardless of which leg affected to optimize ankle function.

\section{Conflicts of interest}

There is no conflict of interest. 


\section{References}

1. Adam NM. Experimental analysis of mechanical properties of selected takraw balls in Malaysia. Malaysian Journal of Movement, Health \& Exercise, 2014;3. https://doi.org/10.15282/mohe.v3i0.18

2. Rezaei M, Mimar R, Azad A. Comparison of Static and Dynamic Balance in Sepak takraw Male Elite Players. International Journal of Basic Sciences \& Applied Research, 2013;2(3):359-62.

3. Jawis M, Singh R, Singh H, Yassin M. Anthropometric and physiological profiles of sepak takraw players. British Journal of Sports Medicine, 2005;39(11):825-9. https://doi.org/10.1136/bjsm.2004.016915

4. Kubo Y, Nakazato K, Koyama K, Tahara Y, Funaki A, Hiranuma K. The relation between hamstring strain injury and physical characteristics of Japanese collegiate Sepak Takraw Players. International Journal of Sports Medicine, 2016;37(12):986-91. https://doi.org/10.1055/s-0042-114700

5. Gribble PA, Hertel J, Plisky P. Using the Star Excursion Balance Test to assess dynamic postural-control deficits and outcomes in lower extremity injury: a literature and systematic review. Journal of Athletic Training, 2012;47(3):339-57. https://doi.org/10.4085/1062-6050-47.3.08

6. Al-Mohrej OA, Al-Kenani NS. Chronic ankle instability: Current perspectives. Avicenna Journal of Medicine, 2016;6(4):103. https://doi.org/10.4103/2231-0770.191446

7. Basnett CR, Hanish MJ, Wheeler TJ, Miriovsky DJ, Danielson EL, Barr J, et al. Ankle dorsiflexion range of motion influences dynamic balance in individuals with chronic ankle instability. International Journal of Sports Physical Therapy, 2013;8(2):121.

8. Jain H, Rathod SA. Correlation between balance and ankle range of motion in community dwelling women having fear of fall aged 60 to 80 years. Journal of Exercise Science and Physiotherapy, 2015;11(1):22-8. https://doi.org/10.18376//2015/v11i1/67092

9. Kosik KB, Johnson NF, Terada M, Thomas AC, Mattacola CG, Gribble PA. Decreased dynamic balance and dorsiflexion range of motion in young and middleaged adults with chronic ankle instability. Journal of Science and Medicine in Spor, 2019;22(9):976-80. https://doi.org/10.1016/j.jsams.2019.05.005

10.Baumbach SF, Brumann M, Binder J, Mutschler $\mathrm{W}$, Regauer $\mathrm{M}$, Polzer $\mathrm{H}$. The influence of knee position on ankle dorsiflexion-a biometric study. BMC Musculoskeletal Disorders, 2014;15(1):1-7. https://doi.org/10.1186/1471-2474-15-246

11. Tavares $\mathrm{P}$, Landsman V, Wiltshire L. Intra-examiner reliability of measurements of ankle range of motion using a modified inclinometer: a pilot study. The Journal of the Canadian Chiropractic Association, 2017;61(2):121.

12. Menadue C, Raymond J, Kilbreath SL, Refshauge KM, Adams R. Reliability of two goniometric methods of measuring active inversion and eversion range of motion at the ankle. BMC Musculoskeletal Disorders, 2006;7(1):1-8. https://doi.org/10.1186/1471-2474-7-60

13. Beazell JR, Grindstaff TL, Sauer LD, Magrum EM, Ingersoll $\mathrm{CD}$, Hertel J. Effects of a proximal or distal tibiofibular joint manipulation on anklerange ofmotionand functional outcomes in individuals with chronic ankle instability. Journal of Orthopaedic \& Sports Physical Therapy, 2012;42(2):125-34. https://doi.org/10.2519/jospt.2012.3729
14.Hoch MC, Staton GS, McKeon JMM, Mattacola CG, McKeon PO. Dorsiflexion and dynamic postural control deficits are present in those with chronic ankle instability. Journal of Science and Medicine in Sport, 2012;15(6):574-9. https://doi.org/10.1016/j.jsams.2012.02.009

15.Plante JE, Wikstrom EA. Differences in clinician-oriented outcomes among controls, copers, and chronic ankle instability groups. Physical Therapy in Sport, 2013;14(4):221-6. https://doi.org/10.1016/j.ptsp.2012.09.005

16.Cordova ML, Sefton JM, Hubbard TJ. Mechanical joint laxity associated with chronic ankle instability: a systematic review. Sports Health, 2010;2(6):452-9. https://doi.org/10.1177/1941738110382392

17.Roach KE, Foreman KB, Barg A, Saltzman CL, Anderson AE. Application of high-speed dual fluoroscopy to study in vivo tibiotalar and subtalar kinematics in patients with chronic ankle instability and asymptomatic control subjects during dynamic activities. Foot \& Ankle International, 2017;38(11):1236-48. https://doi.org/10.1177/1071100717723128

18.Moisan G, Descarreaux M, Cantin V. Effects of chronic ankle instability on kinetics, kinematics and muscle activity during walking and running: a systematic review. Gait \& Posture, 2017;52:381-99. https://doi.org/10.1016/j.gaitpost.2016.11.037

19.Monaghan K, Delahunt E, Caulfield B. Ankle function during gait in patients with chronic ankle instability compared to controls. Clinical Biomechanics, 2006;21(2):168-74. https://doi.org/10.1016/j.clinbiomech.2005.09.004

20.Hertel J, Corbett RO. An updated model of chronic ankle instability. Journal of Athletic Training, 2019;54(6):572-88. https://doi.org/10.4085/1062-6050-344-18

21.Simpson JD, Rendos NK, Stewart EM, Turner AJ, Wilson SJ, Macias DM, et al. Bilateral spatiotemporal postural control impairments are present in participants with chronic ankle instability. Physical Therapy in Sport, 2019;39:1-7. https://doi.org/10.1016/j.ptsp.2019.06.002

22. Vomacka MM, Calhoun MR, Lininger MR, Ko J. Dorsiflexion range of motion in copers and those with chronic ankle instability. International Journal of Exercise Science, 2019;12(1):614.

23.Terada M, Harkey MS, Wells AM, Pietrosimone BG, Gribble PA.Theinfluence ofankledorsiflexionand self-reported patient outcomes on dynamic postural control in participants with chronic ankle instability. Gait\& Posture, 2014;40(1):193-197. https://doi.org/10.1016/j.gaitpost.2014.03.186

24.Olmsted LC, Carcia CR, Hertel J, Shultz SJ. Efficacy of the star excursion balance tests in detecting reach deficits in subjects with chronic ankle instability. Journal of Athletic Training, 2002;37(4):501.

25.Khuman R, Surbala L, Kamlesh T. Dynamic Postural Control Assessment with Star Excursion Balance Test among Chronic Ankle Instability and Healthy Asymptomatic Participants. International Journal of Health and Rehabilitation Sciences, 2014;3(2):55-64. https://doi.org/10.5455/ijhrs.000000053

26. Melam GR, Alhusaini AA, Perumal V, Buragadda S, Kaur $\mathrm{K}$. Comparison of static and dynamic balance between football and basketball players with chronic ankle instability. Saudi Journal of Sports Medicine, 2016;16(3):199. https://doi.org/10.4103/1319-6308.187557

27.Sousa AS, Leite J, Costa B, Santos R. Bilateral proprioceptive evaluation in individuals with unilateral chronic ankle instability. Journal of Athletic Training, 2017;52(4):360-7. https://doi.org/10.4085/1062-6050-52.2.08 
28.Ward S, Pearce AJ, Pietrosimone B, Bennell K, Clark R, Bryant AL. Neuromuscular deficits after peripheral joint injury: a neurophysiological hypothesis. Muscle \& Nerve, 2015;51(3):327-32. https://doi.org/10.1002/mus.24463
29.Hall EA, Chomistek AK, Kingma JJ, Docherty CL. Balanceand strength-training protocols to improve chronic ankle instability deficits, part II: Assessing patient-reported outcome measures. Journal of Athletic Training, 2018;53(6):578-83. https://doi.org/10.4085/1062-6050-387-16

\section{Information about the authors:}

Jessica Phan Zhi Xin; https://orcid.org/0000-0003-3628-3535; jessphan22@gmail.com; INTI International University; Nilai, Malaysia.

Rajkumar Krishnan Vasanthi; (corresponding author); https://orcid.org/0000-0001-6866-8224; rajkumarhari@yahoo.co.in; INTI International University; Nilai, Malaysia.

Vinosh K. Purushothaman; https://orcid.org/0000-0001-5213-3065; vinoshmpt@yahoo.com; INTI International University; Nilai, Malaysia.

Ali Md. Nadzalan; https://orcid.org/0000-0002-0621-2245; ali.nadzalan@fsskj.upsi.edu.my; Sultan Idris Education University; Tanjong Malim, Malaysia.

Cite this article as:

Zhi Xin JP, Vasanthi R.K, Purushothaman VK, Nadzalan A.M. Ankle range of motion and dynamic balance in recreational Sepak Takraw players with and without ankle injury - a comparative study. Pedagogy of Physical Culture and Sports, 2021;25(6):355-

360.

https://doi.org/10.15561/26649837.2021.0603

This is an Open Access article distributed under the terms of the Creative Commons Attribution License, which permits unrestricted use, distribution, and reproduction in any medium, provided the original work is properly cited (http://creativecommons.org/licenses/by/4.0/deed.en).

Received: 05.09.2021

Accepted: 06.10.2021; Published: 30.12.2021 